\title{
Localization in an anisotropic planar aggregate of spheres
}

\author{
Luigi La Ragione ${ }^{1, \star}$, Vincent C. Prantil ${ }^{2}$, and James T. Jenkins ${ }^{3}$ \\ ${ }^{1}$ Dipartimento di Scienze dell'Ingegneria Civile e dell'Architettura, Politecnico di Bari \\ ${ }^{2}$ Department of Mechanical Engineering, Milwaukee School of Engineering 53202 Milwaukee, WI, USA \\ ${ }^{3}$ School of Civil and Environmental Engineering, Cornell University 14853 Ithaca, NY, USA
}

\begin{abstract}
We present a micro-mechanical model that is able to predict localization in a sheared planar aggregate of spheres. We assume a non-linear contact law between interacting particles that deform differently from an affine deformation. Equilibrium determines this deviation. The aggregate is isotropically compressed and then sheared so anisotropy develops because of contacts deletion and a non-linear contact law. Because of anisotropy and fluctuations in particles deformation, the resulting macroscopic stiffness tensor, which relates increments in the average stress with increments in the average strain, is characterized by a lack of major symmetry, $\mathcal{A}_{i j k l} \neq \mathcal{A}_{k l i j}$. At given shear strain and coordination number it is possible to detemine a plane over which discontinuity in strain occurs; this is identified as localization.
\end{abstract}

\section{Introduction}

The authors have recently proposed a micro-mechanical model that highlights a possible mechanism for localization in granular materials [1]. Following previous work [2-4], we identify localization with the possibility of a discontinuity in a component of the incremental strain at a predicted value of the shear strain and a predicted orientation relative to the axis of greatest compression. This is equivalent to a solution for the zero eigenvalue of the acoustic tensor associated with the macroscopic stiffness tensor of the aggregate [5]. In general this condition is possible when the stiffness tensor, that relates increments in the average stress with increments in the average strain, exhibits a lack of major symmetry, $\mathcal{A}_{i j k l} \neq \mathcal{A}_{k l i j}$. This seems a natural state for a granular material and we recover this condition without any hypothesis a priori [6, 7]. We simply require that particles should move according to an average strain and a fluctuation that satisfy equilibrium, with the simultaneous presence of anisotropy. Then a macroscopic stiffness tensor results with no major symmetry. This occurs when the incremental response of the aggregate, at relatively high shear stress, is essentially frictionless and the incremental contribution associated with the tangential force is almost negligible [8].

\section{Theory}

In this paper, we consider a random aggregate of identical particles with diameter $D$ that has been compressed and then sheared. We assume a non-linear contact law with a normal force that follows the Hertz relation between the overlapping and the contact, and a tangential force that is

\footnotetext{
^e-mail: luigi.laragione@poliba.it
}

bilinear with an elastic resistance followed by frictional sliding. The aggregate is axially compressed at constant pressure and, as the shear strain increases, anisotropy develops because of contact deletion and because the normal component of the contact force varies with the orientation of the pair with respect to the direction of greatest compression. The deviatoric stress, $\mathrm{q}$, also increases and both numerical simulations [8] and theoretical models [9] show that it is possible to distinguish a contribution to the deviatoric stress associated with the normal component of the contact force, $q^{N}$, from that associated with the tangential force, $q^{T}$. The result of both numerical simulations and theory is that, close to the stress peak, the deviatoric stress is prescribed almost entirely by $q^{N}$ (e.g. [8], fig. 4). Therefore, in the regime of deformation that preceeds the peak, we assume the aggregate to be in an anisotropic stressed state, incrementally frictionless (because $q^{T}$ does not changes) under a given coordination number, $\hat{k}$ (the average number of contacts per particle).

When an average deformation is applied over a random aggregate, a particle's motion needs to deviate from the average in order to achieve equilibrium. The result is a rather complex system in which particle's motion is described by an average and a fluctuation. Here we work in a simpler situation. We consider a typical pair of spheres $A$ and $B$. Following $[10,11]$, the increment in the relative displacement, $\dot{\mathbf{u}}^{(B A)}$, between contacting spheres $A$ and $B$ is assumed to be the sum of the average and a fluctuation:

$$
\dot{u}_{i}^{(B A)}=\dot{E}_{i j} d_{j}^{(B A)}+\dot{\Delta}_{i}^{(B A)},
$$

where $\dot{\mathbf{E}}$ is the increment in average strain and $\dot{\boldsymbol{\Delta}}^{(B A)}$ is the increment in the difference of the fluctuations in displacements. Unlike the pair $A-B$, the neighbor particles in contact with the pair $A-B$, say $n$, move according to the 


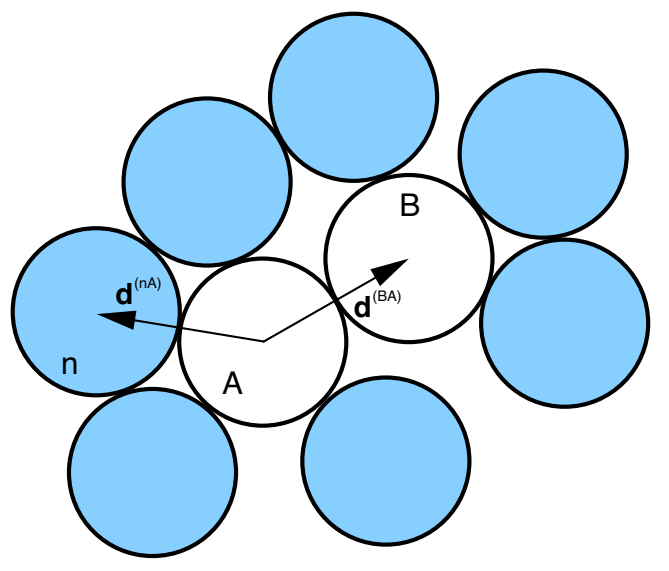

Figure 1. Pair $A-B$ and the neighbor particles $n$.

average strain (see Fig. 1):

$$
\dot{u}_{i}^{(n A)}=\dot{E}_{i j} d_{j}^{(n A)}+\frac{1}{2} \dot{\Delta}_{i}^{(B A)}-\frac{1}{2} \dot{\Sigma}_{i}^{(B A)},
$$

where $\dot{\Sigma}_{j}^{(B A)}$ is the increment in the sum of the fluctuations in displacements. Because the aggregate is seen incrementally frictionless, the incremental contact force between the two contacting spheres, $A$ and $B$, is along their line of centers:

$$
\dot{F}_{i}^{(B A)}=K_{N}^{(B A)} \hat{d}_{i}^{(B A)} \hat{d}_{j}^{(B A)} \dot{u}_{j}^{(B A)},
$$

in which

$$
K_{N}^{(B A)}=\frac{G D^{1 / 2}}{(1-v)}\left(\delta^{(B A)}\right)^{1 / 2},
$$

where $\delta^{(B A)}$ is the inter-particle overlap, $G$ and $v$ are, respectively, the shear modulus and the Poisson ratio of the material of the spheres, and $\hat{\mathbf{d}}^{(B A)}=(\sin \theta, \cos \theta)$ is the unit vector from the center of $A$ to the center of $B$. We assume that the normal component of the contact displacement, $\delta^{(B A)}$, present in the contact stiffness, Eq. (4), depends only on the average strain [12] :

$$
\delta^{(B A)}=-D E_{i j} \hat{d}_{i}^{(B A)} \hat{d}_{j}^{(B A)} .
$$

In the assumed biaxial compression, the area strain $\lambda$ (taken positive for a decrease in area) and the shear strain $\gamma$ are, respectively:

$$
\lambda=-\left(e_{11}+e_{22}\right)
$$

and

$$
\gamma=-\frac{1}{2}\left(e_{22}-e_{11}\right) .
$$

Then, according to Eq. (5), the normal component of the contact displacement can be written as

$$
\delta^{(B A)}=\frac{D}{2}\left(\lambda-2 \gamma+4 \gamma \cos ^{2} \theta\right) .
$$

\subsection{Equilibrium}

At given stressed state, the equations of force equilibrium for sphere $A$ are [10]

$$
\begin{aligned}
\frac{1}{2} A_{i j}^{(B A)}\left(\dot{\Delta}_{j}^{(B A)}-\dot{\Sigma}_{j}^{(B A)}\right) & +\frac{1}{2} K_{N}^{(B A)} \hat{d}_{i}^{(B A)} \hat{d}_{j}^{(B A)}\left(\dot{\Delta}_{j}^{(B A)}+\dot{\Sigma}_{j}^{(B A)}\right) \\
= & -D J_{i j k}^{(B A)} \dot{E}_{j k}
\end{aligned}
$$

with

$$
A_{i j}^{(B A)} \equiv \sum_{n=1}^{N^{(A)}} K_{N}^{(n A)} \hat{d}_{i}^{(n A)} \hat{d}_{j}^{(n A)},
$$

where $N^{(A)}$ is the number of spheres in contact with $A$, and

$$
J_{i j k}^{(B A)} \equiv \sum_{n=1}^{N^{(A)}} K_{N}^{(n A)} \hat{d}_{i}^{(n A)} \hat{d}_{j}^{(n A)} \hat{d}_{k}^{(n A)} .
$$

We employ $\mathbf{d}^{(A B)}=-\mathbf{d}^{(B A)}$ and interchange $A$ and $B$ to obtain the force equilibrium equations for sphere $B$.

The solutions of these local equilibrium equations, up to an error of $1 / \hat{k}$, are $\Delta^{(B A)}$ and $\Sigma^{(B A)}$. The former is our interest,

$$
\dot{\Delta}_{i}^{(B A)}=-D\left[\left(A_{i j}^{(B A)}\right)^{-1} J_{j k m}^{(B A)}-\left(A_{i j}^{(A B)}\right)^{-1} J_{j k m}^{(A B)}\right] \dot{E}_{k m} .
$$

The local solutions depend upon the local neighborhood of each pair. Also, both $\mathbf{J}$ and $\mathbf{A}$ depend on the contact stiffness $K_{N}$ through Eqs. (4) and (8).

Tensors $\mathbf{A}^{(B A)}, \mathbf{A}^{(A B)}, \mathbf{J}^{(B A)}$ and $\mathbf{J}^{(A B)}$ are based on the existence of the contact between $A$ and $B$, and, as a consequence, they depend on $\mathbf{d}^{(A B)}$ or $\mathbf{d}^{(B A)}$. Average values of these sums and differences of the fluctuations result from averaging over the neighborhoods of pairs with nearly the same orientation. Because the averages depend upon the existence of a contact, we refer to them as conditional averages. We denote them with an over-bar and we have

$$
\begin{gathered}
\overline{\dot{\Delta}_{j}^{(B A)}}=-2 d \overline{\left(A_{j i}^{(B A)}\right)^{-1} J_{i m n}^{(B A)}} \dot{E}_{m n}, \\
\overline{\dot{\Sigma}_{j}^{(B A)}}=0 .
\end{gathered}
$$

Again following [10, 11]

$$
\begin{aligned}
& \overline{J_{i j k}^{(B A)}} \equiv K_{N}\left[\beta_{1} \hat{d}_{i}^{(B A)} \hat{d}_{j}^{(B A)} \hat{d}_{k}^{(B A)}\right. \\
& \left.+\beta_{2}\left(\delta_{i k} \hat{d}_{j}^{(B A)}+\delta_{i j} \hat{d}_{k}^{(B A)}+\delta_{k j} \hat{d}_{i}^{(B A)}\right)\right], \\
& \overline{A_{i j}^{(B A)}}=\beta_{3} K_{N} \delta_{i j}
\end{aligned}
$$

with

$$
\begin{gathered}
\beta_{1}=1+\frac{2}{3} g-\rho \frac{2}{3} \\
\beta_{2}=\rho\left(\frac{2}{3}-\frac{\sqrt{3}}{4}\right)-\frac{2}{3} g
\end{gathered}
$$

with

$$
\rho=\frac{3(k-2)}{2 \pi} \text { and } g=\frac{k}{2 \pi},
$$


while

$$
\beta_{3}=\frac{1}{3}+\rho\left(\frac{\sqrt{3}}{12}+\frac{\pi}{6}\right)+g \frac{\pi}{2} .
$$

Making the simplifying assumption that the average of a product is the product of the averages, the average of the difference in fluctuations in translation is given by

$$
\overline{\dot{\Delta}_{i}^{(B A)}}=D R_{i j k} \dot{E}_{j k},
$$

with

$R_{i j k}=\alpha_{1} \hat{d}_{i}^{(B A)} \hat{d}_{j}^{(B A)} \hat{d}_{k}^{(B A)} \dot{E}_{j k}+\alpha_{2}\left(\delta_{i j} \hat{d}_{k}^{(B A)}+\delta_{i k} \hat{d}_{j}^{(B A)}+\delta_{k j} \hat{d}_{i}^{(B A)}\right) \dot{E}_{j k}$

where

$$
\alpha_{1}=-2 \beta_{3}^{-1} \beta_{1} \text { and } \alpha_{2}=-2 \beta_{3}^{-1} \beta_{2} .
$$

With Eq. (16), Eq. (1) can be written as

$$
\overline{\dot{u}_{i}^{(B A)}}=D\left(1+2 \alpha_{2}\right) \dot{E}_{i j} \hat{d}_{j}^{(B A)}+D\left(\alpha_{1} \hat{d}_{j}^{(B A)} \dot{E}_{j k} \hat{d}_{k}^{(B A)}+\alpha_{2} \dot{E}_{k k}\right) \hat{d}_{i}^{(B A)} \text {. }
$$

\section{Stiffness tensor}

The incremental average stress is

$$
\dot{\sigma}_{i j}=\frac{n D \hat{k}}{\pi} \int_{0}^{\theta_{1}} \dot{F}_{i} \hat{d}_{j} d \theta
$$

where the integration is limited to contacting pairs, the distribution function is assumed isotropic, $\hat{k} /(2 \pi)$ and $n$ is the number of particles per unit area. For symmetry condition we have restricted our attention to the range $0 \leq \theta \leq \theta_{1}$, with $\theta_{1} \leq \pi / 2$. The upper limit in the integral refers to the deletion angle, $\theta_{1}$, the angle associated with $\delta^{(B A)}=0$,

$$
\theta_{1}=\cos ^{-1} \sqrt{\frac{2 \gamma-\lambda}{4 \gamma}}
$$

That is, the deletion angle is the orientation of the normal component of the contact force that relaxes to zero which corresponds to the vanishing of the contact stiffness.

Under isotropic pressure, fluctuations play a minor role [10]; therefore the condition of constant pressure is developed with the simple average strain hypothesis and we obtain for $\dot{p}=0$ the following expression

$$
\int_{0}^{\theta_{1}}\left(\lambda-2 \gamma+4 \gamma \cos ^{2} \theta\right)^{1 / 2}\left(\dot{\lambda}-2 \dot{\gamma}+4 \dot{\gamma} \cos ^{2} \theta\right) d \theta=0 .
$$

We integrate with Simpson's rule to derive a relation between the area strain $\lambda$ and the shear strain $\gamma$.

From Eq. (18), with the incremental contact force given by Eq. (3) and the incremental displacement given by eq. (17), we obtain

$$
\begin{array}{ll}
\dot{\sigma}_{i j} & =\omega\left(1+\alpha_{1}+2 \alpha_{2}\right) \\
\times & \int_{0}^{\theta_{1}}\left(\lambda-2 \gamma+4 \gamma \cos ^{2} \theta\right)^{1 / 2} \hat{d}_{k} \hat{d}_{m} \hat{d}_{i} \hat{d}_{j} \dot{E}_{k m} d \theta \\
+\quad \omega \alpha_{2} \int_{0}^{\theta_{1}}\left(\lambda-2 \gamma+4 \gamma \cos ^{2} \theta\right)^{1 / 2} \hat{d}_{i} \hat{d}_{j} \delta_{k m} \dot{E}_{k m} d \theta .
\end{array}
$$
with

where $\omega=n D^{3} \hat{k} G /[(1-v) \pi \sqrt{2}]$. Anisotropy is present in the stiffness and in the limit of integration where the integrals are extened to $\theta_{1}$ which varies as the shear strain increases, Eq. (19). In a more compact form we have

$$
\dot{\sigma}_{i j}=\mathcal{A}_{i j k m} \dot{E}_{k m},
$$

where, with $\mathbf{h}$ the vertical axes of compression,

$$
\begin{array}{cl}
\mathcal{A}_{i j k m} & =\eta_{1} h_{m} h_{k} h_{j} h_{i}+\eta_{2} \delta_{i j} \delta_{k m}+\eta_{3}\left(\delta_{i k} \delta_{j m}+\delta_{i m} \delta_{j k}\right) \\
+ & \eta_{4} \delta_{k m} h_{i} h_{j}+\eta_{5} h_{m} h_{k} \delta_{i j} \\
+ & \eta_{6}\left(\delta_{i k} h_{j} h_{m}+\delta_{j m} h_{k} h_{i}+\delta_{i m} h_{k} h_{j}+\delta_{j k} h_{i} h_{m}\right),
\end{array}
$$

$$
\begin{aligned}
& \eta_{1}=\omega\left(1+\alpha_{1}+2 \alpha_{2}\right) a_{1}, \\
& \eta_{2}=\omega\left(1+\alpha_{1}+2 \alpha_{2}\right) \frac{a_{2}}{3}+\omega \alpha_{2} a_{3} \\
& \eta_{3}=\omega\left(1+\alpha_{1}+2 \alpha_{2}\right) \frac{a_{2}}{3}, \\
& \eta_{4}=\omega\left(1+\alpha_{1}+2 \alpha_{2}\right)\left(a_{4}-\frac{a_{2}}{3}\right)+\omega \alpha_{2} a_{5}, \\
& \eta_{5}=\omega\left(1+\alpha_{1}+2 \alpha_{2}\right)\left(a_{4}-\frac{a_{2}}{3}\right),
\end{aligned}
$$

and

$$
\eta_{6}=\omega\left(1+\alpha_{1}+2 \alpha_{2}\right)\left(a_{4}-\frac{a_{2}}{3}\right),
$$

in which,

$$
\begin{aligned}
a_{1} & \equiv \int_{0}^{\theta_{1}}\left(\lambda-2 \gamma+4 \gamma \cos ^{2} \theta\right)^{1 / 2} \\
& \times\left(\cos ^{4} \theta-6 \sin ^{2} \theta \cos ^{2} \theta+\sin ^{4} \theta\right) d \theta, \\
a_{2} & \equiv \int_{0}^{\theta_{1}}\left(\lambda-2 \gamma+4 \gamma \cos ^{2} \theta\right)^{1 / 2} \sin ^{4} \theta d \theta, \\
a_{3} & \equiv \int_{0}^{\theta_{1}}\left(\lambda-2 \gamma+4 \gamma \cos ^{2} \theta\right)^{1 / 2} \sin ^{2} \theta d \theta, \\
a_{4} & \equiv \int_{0}^{\theta_{1}}\left(\lambda-2 \gamma+4 \gamma \cos ^{2} \theta\right)^{1 / 2} \sin ^{2} \theta \cos ^{2} \theta d \theta, \\
a_{5} & \equiv \int_{0}^{\theta_{1}}\left(\lambda-2 \gamma+4 \gamma \cos ^{2} \theta\right)^{1 / 2} \cos 2 \theta d \theta .
\end{aligned}
$$

\section{Localization condition}

We investigate the possibility of a discontinuity in a component of the incremental strain over a band with unit normal l. Following $[2,3]$ we have

$$
\operatorname{det}\left|l_{i} \mathcal{A}_{i j p q} l_{q}\right|=0 .
$$

With $\mathbf{I}=(\cos \psi, \sin \psi)$, and the stiffness tensor given by Eq. (23), the localization condition corresponds to

$$
0=Z_{1} \cos ^{4} \psi+Z_{2} \cos ^{2} \psi+Z_{3}
$$

with

$$
\begin{aligned}
Z_{1} & =\mathcal{A}_{1212}\left(\mathcal{A}_{1122}+\mathcal{A}_{2211}+\mathcal{A}_{1111}+\mathcal{A}_{2222}\right) \\
& +\mathcal{A}_{1122} \mathcal{A}_{2211}-\mathcal{A}_{2222} \mathcal{A}_{1111}, \\
Z_{2} & =\mathcal{A}_{2222} \mathcal{A}_{1111}-\mathcal{A}_{1212}\left(\mathcal{A}_{1122}+\mathcal{A}_{2211}+2 \mathcal{A}_{2222}\right) \\
& -2 \mathcal{A}_{1122} \mathcal{A}_{2211}, \\
Z_{3} & =\mathcal{A}_{2222} \mathcal{A}_{2112} .
\end{aligned}
$$


Table 1. Localization angle at given coordination number and normalized shear strain

\begin{tabular}{lll}
\hline$\hat{k}$ & $\psi(\operatorname{deg})$ & $\gamma / \lambda_{0}$ \\
\hline 5 & 16.78 & 2.77 \\
4.5 & 18.33 & 2.67 \\
4 & 20.60 & 2.44 \\
3.5 & 23.70 & 2.25 \\
3 & 28.57 & 1.65 \\
2.5 & 38.35 & 0.83 \\
\hline
\end{tabular}

With $m=\cos ^{2} \psi$ we have

$$
m_{1 / 2}=\frac{-Z_{2} \pm \sqrt{Z_{2}^{2}-4 Z_{3} Z_{1}}}{2 Z_{1}} .
$$

The components of the stiffness tensor which involve the knowledge of integrals $a_{1}, a_{2}, . . a_{5}$ are determined by means of Simpson's rule. At lower shear strain the discrimant is negative in the range of coordination number between 6 and 2. Therefore we determine the value of the strain for which a real value of the angle is first possible; this occurs when first the discrimant $Z_{2}^{2}-4 Z_{1} Z_{3}$ vanishes. There,

$$
\cos \psi=\sqrt{\frac{-Z_{2}}{2 Z_{1}}}
$$

Results are reported in Table 1.

\section{Conclusion}

While it is well-known that localization in granular materials is associated with the solution of the vanishing of the acoustic tensor related to the stiffness tensor of the aggregate, it is less obvious why the stiffness tensor should not be characterized by a major symmetry. In [2], this condition is physically justified by the non-associative flow rule for an elasto-plastic material with a yield surface characterized by a vertex structure. In [4], it is not assumed a priori and the numerical micro-mechanical approach seems to support this condition, although the authors refer again to the loss of the plastic normality. In [13], in the context of a micro-mechanical model, when particles are constrained to move with the average strain, it is possible to predict a potential function different from the yield function and so a non-associative flow. Nevertheless the same model, because of the absence of fluctuation, does not predict a loss of symmetry in the stiffnness tensor and therefore no localization. Here we show that it is anisotropy and fluctuations, two key mechanisms in granular materials, that provide a structure to the macroscopic stiffness tensor that leads to the possibility of the vanishing of the acoustic tensor, for a given coordination number, at a given shear strain. Numerical simulations may be a tool to understand which quantities are crucial to obtain the loss of major symmetry in the stiffness tensor and how fluctuations, anisotropy are associated with plastic normality.

\section{Acknowledgements}

L. La Ragione is grateful to Office of Naval Research under the grant N62909-17-1-2048 and GNFM (Italy) to support his research.

\section{References}

[1] La Ragione L., Prantil, V.C., Jenkins J.T., Journal of the Mechanics of Physics and Solids, 83, 46-159 (2015)

[2] Rudnicki, J.W. and Rice, J. R. Journal of the Mechanics and Physics of Solids 23, 371-394 (1975).

[3] Vardoulakis, I., Mechanics Research Communication 3, 209-214 (1976)

[4] Wren, J.R., Borja, R.I., Computer Methods in Applied Mechanics and Engineering 141, 221-246 (1997)

[5] Hill, R., Journal of the Mechanics of Physics and Solids, 10, 1-16 (1962)

[6] La Ragione, L., Oger, L., Recchia, G., Sollazzo, A., Proceedings of the Royal Society A: Mathematical, Physical and Engineering Sciences,471, 13-31 (2015)

[7] La Ragione, L., Journal of the Mechanics of Physics and Solids, 95, 147-168 (2016)

[8] Thornton, C. and Antony, S.J., Philosophical Transactions of the Royal Society of London Series A 356, 2763-2782 (1998)

[9] Jenkins, J. T. and Strack, O.D.L., Mechanics of Materials 16, 25-33 (1993).

[10] Jenkins, J. T., Johnson, D. L., La Ragione, L. and Makse, H., Journal of the Mechanics and Physics of Solids 53, 197-225 (2005)

[11] Jenkins, J.T., Agnolin, I., La Ragione, L., Mechanics of Materials 38, 687-701 (2006)

[12] Kuhn, M., Mechanics of Materials, 42, 827-840 (2010)

[13] La Ragione, L., Prantil, V.C. and Sharma, I., International Journal of Plasticity 24, 168-189 (2008). 